\title{
LGBTQI+ allyship in academia
}

Standfirst: Come out, come out, wherever you are: the geosciences need LGBTQI+ allies, says Anson Mackay.

My love of the geosciences started with an introduction to palaeoecology as part of my undergraduate degree in Botany. It's fair to say that palaeoecology has been fundamental to my life ever since. It has allowed me to develop critical perspectives on long-term change in planetary ecosystems, participate in workshops and conferences around the world, and to educate new generations of geoscientists. But while the world of geoscience always had time for me as a scientist, it often didn't have time for me as someone who did not fit expected norms - someone who refused to hide that they were gay.

Like other under-represented minorities, I've experienced name-calling, harassment, exclusion, and even the threat of physical violence whilst carrying out my work as a geoscientist. My story is not unique. Across STEM, LGBTQI+ people (the diverse community of lesbian, gay, bisexual, transgender, queer and intersex people, as well as others who have marginalized sexual orientations, gender identities and gender expressions) are more likely to feel devalued at work and to experience harassment and blocks in their careers, leading to greater health issues and to an increased likelihood of leaving STEM altogether. To change this culture, we need active intervention from non LGBTQI+ geoscientists; in essence, we need allies.

So, what is allyship? Allyship is when an individual, a committee, a network, or an organisation, through conscious effort, promotes a culture of inclusion, but does not seek recognition or credit for having done so. Good allyship is an ever-evolving act, not a one-off performance, and has the potential to greatly effect minoritized communities as a whole, not only those who are LGBTQI+.

Being a good ally also means thinking about one's own background and the possible advantages (or privileges) that come with that. Acknowledging such advantages does not take away from anyone's own struggles, but it can help to frame the challenges and barriers faced by others. These advantages equally apply to LGBTQI+ people, where one aspect of their identity might give them advantages but other aspects might bring disadvantages. For instance, although I've experienced direct and indirect homophobia, as a white, cis, able-bodied man, the discrimination I have faced will be different than that faced by women, people of colour, people who are transgender or non-binary, and people who are disabled. Therefore, I need to be an ally for those people. Here, I outline some of the specific barriers LGBTQI+ geoscientists face, pinpointing ways in which individuals, Learned Societies and organisations can actively do things differently to be good allies.

\section{[H1] Self-education}

Heterosexuality and the gender binary are assumed as the norm. These assumptions lead to increased levels of discrimination and harassment of LGBTQI+ people, and ultimately their exclusion, unless they downplay their gender identity, sexual orientation or gender expression to fit in. A strong act of allyship is to therefore take time to learn about the social power imbalances and structural inequalities that LGBTQI+ geoscientists face.

Other acts of allyship are often simple, but can have large impact. For example, respect the pronouns that people use for themselves, and encourage others to add their pronouns to email signatures and social media bios. Normalising pronoun use has several advantages, including creating an environment where different gender identities are accepted, allowing transgender and 
non-binary people to feel secure and to bring their whole selves to work. Also, don't assume that everyone is heterosexual! Facilitate conversations on diversity and inclusion during regular group meetings, creating an inclusive culture so as to enrich the science we do.

\section{[H1] In the classroom, laboratory or field}

There are a number of different ways that educators can be an LGBTQI+ ally in the classroom, in the laboratory and in the field. Call out derogatory language when you hear it, such as "that's so gay", and make sure that you use inclusive language yourself. Participate in global LGBTQI+ events such as LGBTQ+ STEM Day. At UCL, we have a 'Friends of Out@UCL' network that provides training for LGBTQI+ allies, that also acts to build confidence in identifying and challenging offensive behaviour and language, and to help champion LGBTQI+ equality. I have straight colleagues who have done this training in my department, and at first I was surprised, then a bit over-come, then very proud to realise that I work with straight geoscientists who genuinely took the time to understand better the anxieties and barriers that their LGBTQI+ colleagues and students face.

In the geosciences, visibility of LGBTQI+ people is very low, yet we know that diverse role models are important. Here, allyship can take the form of celebrating the achievements of LGBTQI+ geoscientists and related disciplines, and not straightwashing their contributions, for example in journal clubs and teaching material. At UCL Geography, we set up a LGBTQI+ network called 'Out in Geography', which hosts meetings on a range of topics from gender identity in academia to what are turning into annual LGBTQI+ themed Wikithons, where we create and edit Wikipedia pages for notable LGBTQI+ scientists. The network is led by two LGBTQI+ members of staff, raising the visibility and profile of LGBTQI+ people in the department immediately to all new students who join.

Having codes of conduct applicable to all lab members and visitors, where no harassment of any form is tolerated, including for sexual orientation, gender identity and gender expression, is really important for creating safe and respectful environments. Clear routes of reporting harassment should be given. Encourage all lab members to get involved with diversity and inclusion activities.

Implementing an inclusive risk assessment tool for travel and fieldwork ensures safe fieldwork strategies for all at-risk individuals. However, LGBTQI+ allyship needs particular consideration, as significant numbers of LGBTQI+ people do not feel safe in the field. Avoid, for example taking students on fieldtrips to a jurisdiction which has laws that are actively hostile to LGBTQI+ people.

\section{[H1] Conferences and workshops}

Workshops and conferences are a mainstay of geoscience societies and unions, and making these more inclusive is an absolute must. For LGBTQI+ participants, this can take a number of forms. Like fieldwork above, avoid holding conferences in a jurisdiction which has laws that are actively hostile to LGBTQI+ people. At conferences, have space in the programme for an organised LGBTQI+ social session. Good practice for conferences is to have spaces on name badges for pronouns, and to ensure a diverse range of invited and other speakers in terms of gender and ethnicity (avoid the Manel!).

Good allyship is not easy, and it can take many forms. While visible LGBTQI+ people already established in the geosciences can provide role models, we need our allies to come out and recognise the systemic inequalities we face, and to encourage and empower LGBTQI+ people to feel like they belong. Only then will we have a discipline that is truly inclusive for LGBTQI+ geoscientists. 
Email:ans.mackay@ucl.ac.uk

Pronouns: $\mathrm{He} / \mathrm{Him}$

\section{Competing interests}

The author declares no competing interests. 